\section{Mortalidade por suicídio entre crianças indígenas no Brasil}

\author{
Mortality from suicide in indigenous children \\ in Brazil
}

\section{Mortalidad por suicidio entre niños indígenas \\ en Brasil}

Maximiliano Loiola Ponte de Souza 1

doi: 10.1590/0102-311X00019219

\section{Resumo}

O objetivo deste trabalho foi descrever as características, a distribuição e as taxas de mortalidade por suicídio entre crianças indígenas no Brasil, comparativamente as não indígenas. Realizou-se um estudo descritivo, compreendendo os anos de 2010 a 2014, utilizando dados dos sistemas nacionais de informação. Foram selecionados os óbitos de pessoas com idades de 10 a 14 anos, cuja causa básica foi classificada como "lesões autoprovocadas voluntariamente". $O$ enforcamento foi o meio utilizado com mais frequência, tanto entre indígenas como entre não indígenas, embora tenha sido mais frequente no primeiro grupo. Entre indígenas, a ocorrência de suicídio em hospital ou outros estabelecimentos de saúde foi menor do que o observado entre os não indígenas. Aproximadamente, 3/4 dos suicídios entre crianças indígenas ocorreram em 17 municípios. A taxa de mortalidade por suicídio entre crianças indígenas foi de 11,0/100 mil (8,4-14,3), 18,5 (10,9-31,6) vezes maior do que a observada entre as não indígenas, 0,6/100 mil (0,5-0,6), sem diferenças entre meninos e meninas. Este trabalho evidenciou, pela primeira vez, em escala nacional, especificidades das características do suicídio indígena, suas elevadas taxas, bem como identificou ainda áreas prioritárias para intervenções.

Suicídio; Criança; População Indígena

\author{
Correspondência \\ M. L. P. Souza \\ Fiocruz Ceará, Fundação Oswaldo Cruz. \\ Rua São José s/n, Eusébio, CE 61760-000, Brasil. \\ maxkaelu@hotmail.com \\ 1 Fiocruz Ceará, Fundação Oswaldo Cruz, Eusébio, Brasil.
}




\section{Introdução}

Estudar a mortalidade por suicídio em crianças consiste em um grande desafio. Afinal, crianças tirarem suas próprias vidas parece, para muitos, algo absurdo e inaceitável, indo contra o "paradigma de sonhos e alegrias” 1 (p. 3099) que supostamente deveriam fazer parte de suas vidas. Outro desafio seria definir a partir de quando crianças entenderiam o conceito de morte, e sua irreversibilidade, algo necessário para caracterizar um ato contra si mesmo como o suicídio 2 . Evidências da literatura apontam que, a partir dos oito anos, em média, essa habilidade seria adquirida 3. Considerando a agregação das idades em grupos de cinco anos usualmente realizada nos estudos demográficos e epidemiológicos, autores propõem que se adote a faixa etária de 10 a 14 anos como a faixa etária infantil ou de crianças, quando se estuda o suicídio 4 . Adotar limites inferiores implicaria no risco aumentado de selecionar óbitos de pessoas que poderiam não ter a clareza da irreversibilidade da morte. Estender o limite superior até 19 anos, por exemplo, resultaria na inclusão de pessoas cujas vivências, experiências e percepções de mundo seriam bem diferentes daquelas dos limites mais baixos da faixa etária. Entretanto, nem sempre essa recomendação é seguida, o que dificulta a comparação de achados obtidos em diferentes estudos 1,4.

Apesar de as taxas de mortalidade por suicídio serem baixas entre os mais jovens, em alguns contextos do mundo, elas vêm aumentando 1,4. A despeito desse fato, são poucos os trabalhos que investigam, especificamente, o suicídio entre crianças, e a maioria foi realizada em países desenvolvidos 4 . No Brasil, apesar de termos um corpus relativamente robusto de estudos sobre suicídio, Sousa et al. 1, ao realizarem uma revisão da literatura a respeito do suicídio entre crianças, não conseguiram encontrar um único artigo relevante sobre o tema publicado no país.

Evidências apontam ainda que, em determinadas minorias étnico-raciais, como os indígenas (aborígines ou populações nativas), o suicídio entre crianças apresenta taxas bem mais elevadas do que a observada na população geral 4,5,6. Não obstante esse fato, a maior parte dos estudos sobre suicídio entre indígenas não apresenta sequer dados sobre a população infantil 7, incluindo boa parte das pesquisas nacionais $8,9,10,11,12,13$. Apenas mais recentemente, observa-se uma preocupação explícita com o suicídio entre crianças indígenas no Brasil, embora restrita a um contexto específico 14. Dessa forma, diante dessa lacuna, o objetivo deste artigo foi descrever as características, a distribuição e as taxas de mortalidade por suicídio entre crianças indígenas no Brasil, comparativamente as não indígenas.

\section{Método}

\section{Desenho do estudo}

Estudo descritivo, compreendendo o período de 2010 a 2014.

\section{População do estudo}

Em 2010, segundo o Censo Demográfico 15, a população indígena do Brasil era estimada em 817.963 pessoas, $0,4 \%$ da população total do país. Entre os indígenas, 11,6\% de sua população, 94.826 indivíduos tinham entre 10 e 14 anos, enquanto, entre os não indígenas, esse grupo etário equivalia a 9\% da população. A maior parte da população indígena de 10 a 14 anos residia nas macrorregiões Norte $(42,1 \%)$ e Nordeste (23,9\%), e o restante, nas Centro-oeste (17\%), Sudeste (7,8\%) e Sul (7,8\%). Já a população não indígena de 10 a 14 anos concentrava-se, sobretudo, nas macrorregiões Sudeste $(38,7 \%)$ e Nordeste (30,6\%), seguidas pelas macrorregiões Sul (13,4\%), Norte (10,1\%) e Centro-oeste (7,2\%).

\section{Fonte de dados}

Os dados de mortalidade foram obtidos no Sistema de Informações sobre Mortalidade (SIM) do Ministério da Saúde (http://tabnet.datasus.gov.br/cgi/deftohtm.exe?sim/cnv/ext10uf.def, acessado em 18/Dez/2018). Os dados populacionais foram estimados a partir dos censos oficiais de 2000 e 2010 (Instituto Brasileiro de Geografia e Estatística. https://sidra.ibge.gov.br/pesquisa/censo-demo 
grafico/demografico-2010/inicial, acessado em 18/Dez/2018), por meio de interpolação geométrica anual. No Brasil, os censos investigam a "raça ou cor [da pele]", por meio do critério de autodeclaração, nas seguintes categorias: brancos, pretos, indígenas, amarelos e pardos. As informações a respeito da localização dos municípios nos quais ocorreram suicídios entre crianças indígenas foram obtidas junto ao Instituto Brasileiro de Geografia e Estatística (http://www.ibge.gov.br/home/geociencias/ geografia/, acessado em 15/Dez/2018).

\section{Critérios de inclusão}

Foram selecionados os óbitos de pessoas com idades entre 10 e 14 anos 1,4, cuja causa básica foi classificada como "lesões autoprovocadas voluntariamente" (códigos X60-X84) 16, ocorridos no Brasil, no período de 2010-2014.

\section{Variáveis investigadas}

As variáveis investigadas foram: raça/cor da pele (indígenas e não indígenas, nesta última, incluídos brancos, pretos, pardos e amarelos); sexo (masculino; feminino); local de ocorrência (domicílio, via pública, hospital e outros estabelecimentos de saúde, outros e ignorado); método utilizado para cometer o suicídio (enforcamento; intoxicação; disparo por arma de fogo e outros); macrorregião (Norte, Nordeste, Centro-oeste, Sudeste e Sul) e município de residência.

\section{Mensuração e análise estatística}

Em todas as mensurações realizadas, os casos foram divididos em indígenas e não indígenas e, posteriormente, subdivididos em sexos masculino e feminino.

Para caracterização dos óbitos por suicídio, foram calculadas frequências absolutas e relativas (expressas em percentuais), utilizando as variáveis selecionadas. Já as taxas de mortalidade por suicídio foram calculadas pela razão entre o número de suicídios e a respectiva população, sendo expressão em suicídio/100 mil habitantes. Para esses indicadores, foram também calculados intervalos de $95 \%$ de confiança (IC95\%).

$\mathrm{Na}$ análise das características, considerou-se que havia diferença estatística entre os grupos quando as frequências não apresentavam intersecções, considerando seus intervalos de confiança. Para comparar as taxas de mortalidade entre diferentes grupos, foram calculadas razões de taxas de mortalidade por suicídio, com seus respectivos IC95\%. Admitiu-se diferença estatística entre duas taxas quando a razão entre elas, considerando os intervalos de confiança, não continha o valor um nem apresentava intersecções entre si.

Os casos de suicídio entre indígenas foram georreferenciados no mapa do Brasil, tomando, como referência, o centroide do município de residência da pessoa que faleceu.

\section{Resultados}

Foram registrados 584 suicídios de crianças, desses, 55 [9,4\% (7,0-11,8)] eram indígenas. Conforme a Tabela 1, entre indígenas, houve predomínio de suicídio entre meninas, $58,2 \%(45,1-71,2)$, enquanto, entre não indígenas, foi entre meninos, $60 \%(55,7-64,3)$. O enforcamento foi o meio utilizado com mais frequência, tanto entre indígenas $[96,4 \%(91,4-101,3)]$ como entre não indígenas $[62,8 \%$ (58,6$67,0)]$, embora mais frequente no primeiro grupo. Entre os indígenas, não se observou suicídios por intoxicação ou arma de fogo, diferente do observado entre os não indígenas. Tanto entre os indígenas $[65,5 \%(52,9-78,0)]$ como entre os não indígenas $[54,1 \%(49,7-58,4)]$, a maior parte dos óbitos ocorreu no domicílio. Destaca-se que, entre indígenas, a ocorrência de suicídio em hospital ou outros estabelecimentos de saúde foi menor do que a observada entre os não indígenas, 1,8\% (1,7-5,3) e 30,3\% (26,3-34,3), respectivamente.

Como pode se observar na Tabela 2, entre meninos e meninas indígenas, predominou o suicídio por enforcamento, realizado no domicílio, não havendo diferenças entre eles. Entre os meninos não 
Tabela 1

Características da mortalidade por suicídio entre crianças indígenas e não indígenas. Brasil, 2010-2014.

\begin{tabular}{|c|c|c|}
\hline & $\begin{array}{c}\text { Indígenas ( } \mathrm{n}=55) \\
\%(\mathrm{IC} 95 \%)\end{array}$ & $\begin{array}{c}\text { Não indígenas }(n=529) \\
\%(I C 95 \%)\end{array}$ \\
\hline \multicolumn{3}{|l|}{ Sexo } \\
\hline Masculino & $41,8(28,8-54,9)$ & $60,0(55,7-64,3)$ \\
\hline Feminino & $58,2(45,1-71,2)$ & $40,0(35,7-44,3)$ \\
\hline \multicolumn{3}{|l|}{ Local de ocorrência } \\
\hline Domicílio & $65,5(52,9-78,0)$ & $54,1(49,7-58,4)$ \\
\hline Via pública & $1,8(1,7-5,3)$ & $3,8(2,1-5,4)$ \\
\hline Hospital/Outros estabelecimentos & $1,8(1,7-5,3)$ & $30,3(26,3-34,3)$ \\
\hline Outros & $30,9(18,7-43,1)$ & $11,9(9,1-15,1)$ \\
\hline \multicolumn{3}{|l|}{ Método } \\
\hline Enforcamento & $96,4(91,4-101,3)$ & $62,8(58,6-67,0)$ \\
\hline Intoxicação & - & $16,2(13,0-19,5)$ \\
\hline Arma de fogo & - & $10,5(7,8-13,2)$ \\
\hline Outros & $3,6(1,3-8,6)$ & $10,5(7,8-13,2)$ \\
\hline \multicolumn{3}{|l|}{ Macrorregião } \\
\hline Norte & $49,1(35,9-62,3)$ & $16,8(13,6-20,1)$ \\
\hline Centro-oeste & $45,5(32,3-58,6)$ & $12,3(9,4-15,1)$ \\
\hline Nordeste & $3,6(1,3-8,6)$ & $26,7(22,9-30,6)$ \\
\hline Sudeste & $1,8(1,7-5,3)$ & $27,7(23,8-31,6)$ \\
\hline Sul & - & $16,4(13,2-19,7)$ \\
\hline \multicolumn{3}{|l|}{ Unidade da Federação } \\
\hline Amazonas & $41,8(28,8-54,9)$ & $5,3(3,4-7,3)$ \\
\hline Mato Grosso do Sul & $43,6(30,5-56,7)$ & $3,4(1,8-4,9)$ \\
\hline Outros & $14,5(5,2-23,9)$ & $91,3(88,8-93,7)$ \\
\hline
\end{tabular}

IC95\%: intervalo de 95\% de confiança.

Fonte: Sistema de Informações sobre Mortalidade (SIM) do Ministério da Saúde (http://tabnet.datasus.gov.br/cgi/ deftohtm.exe?sim/cnv/ext10uf.def, acessado em 18/Dez/2018).

\section{Tabela 2}

Características da mortalidade por suicídio entre crianças indígenas e não indígenas, conforme sexo. Brasil, 2010-2014.

\begin{tabular}{|c|c|c|c|c|}
\hline & \multicolumn{2}{|c|}{ Indígenas $(\mathrm{n}=55)$} & \multicolumn{2}{|c|}{ Não indígenas $(\mathrm{n}=529)$} \\
\hline & Masculino $(n=23)$ & Feminino $(n=32)$ & Masculino $(n=317)$ & Feminino $(n=212)$ \\
\hline & \% (IC95\%) & $\%(I C 95 \%)$ & \% (IC95\%) & $\%(I C 95 \%)$ \\
\hline \multicolumn{5}{|l|}{ Local de ocorrência } \\
\hline Domicílio & $78,3(61,4-95,1)$ & $56,3(39,1-73,4)$ & $57,8(52,2-63,2)$ & $48,5(41,6-55,4)$ \\
\hline Via pública & $4,3(-4-12,7)$ & - & $4,3(2,0-6,6)$ & $3,0(0,6-5,3)$ \\
\hline Hospital/Outros estabelecimentos & - & $3,1(-2,9-9,2)$ & $23,4(18,7-28,2)$ & $40,6(33,8-47,4)$ \\
\hline Outros & $17,4(1,9-32,9)$ & $40,6(23,6-57,6)$ & $14,5(10,6-18,5)$ & $7,9(4,2-11,6)$ \\
\hline \multicolumn{5}{|l|}{ Método } \\
\hline Enforcamento & $91,3(79,8-102,8)$ & 100,0 & $71,3(66,2-76,4)$ & $50,0(43,1-56,9)$ \\
\hline Intoxicação & - & - & $5,6(3,0-8,2)$ & $32,2(25,7-38,6)$ \\
\hline Arma de fogo & - & - & $13,5(9,7-17,4)$ & $5,9(2,7-9,2)$ \\
\hline Outros & $8,7(-2,8-20,2)$ & - & $9,6(6,3-12,9)$ & $11,9(7,4-16,3)$ \\
\hline
\end{tabular}

IC95\%: intervalo de 95\% de confiança.

Fonte: Sistema de Informações sobre Mortalidade (SIM) do Ministério da Saúde (http://tabnet.datasus.gov.br/cgi/deftohtm.exe?sim/cnv/ext10uf.def, acessado em 18/Dez/2018). 
indígenas, houve maior frequência de suicídios por enforcamento [71,3\% (66,2-76,4)] e por arma de fogo $[13,5 \%(9,7-17,40)]$, e menor por intoxicação [5,6\% (3,0-8,2)], em comparação as meninas que apresentam valores de 50\% (43,1-56,9), 5,9\% (2,7-9,2) e 32,2\% (25,7-38,6), respectivamente. Entre meninas não indígenas, observou-se uma maior ocorrência de suicídios em hospital e outros estabelecimentos de saúde, em comparação aos meninos, 40,6\% (33,8-47,4) e 23,4\% (18,7-28,2), respectivamente.

A quase totalidade dos suicídios entre indígenas, 94,5\% (88,5-100,5) ocorreu nas macrorregiões Norte e Centro-oeste, enquanto, entre os não indígenas, 29,1\% (25,1-33,1) ocorreram nessas macrorregiões (Tabela 1). Aproximadamente, 3/4 dos suicídios entre crianças indígenas ocorreram em 17 municípios, agrupáveis em três grupos, um localizado no sudoeste do Amazonas [27,3\% (15,5-39,0)], outro no noroeste desse estado [9,1\% (1,5-16,7)] e o outro no sul do Estado do Mato Grosso do Sul $[40 \%(27,1-52,9)]$ (Figura 1).

A taxa de mortalidade por suicídio entre crianças no Brasil foi de 0,7/100 mil (0,6-0,7). Conforme a Tabela 3, entre indígenas, a taxa foi de 11,0/100 mil (8,4-14,3), 18,5 (10,9-31,6) vezes maior do que a observada entre não indígenas, 0,6/100 mil (0,5-0,6). Tanto entre meninos quanto entre meninas, as taxas de mortalidade por suicídio foram maiores entre os indígenas. Não houve diferença estatística entre as taxas de mortalidade por suicídio entre meninos e meninas nos subgrupos de indígenas e não indígenas.

\section{Figura 1}

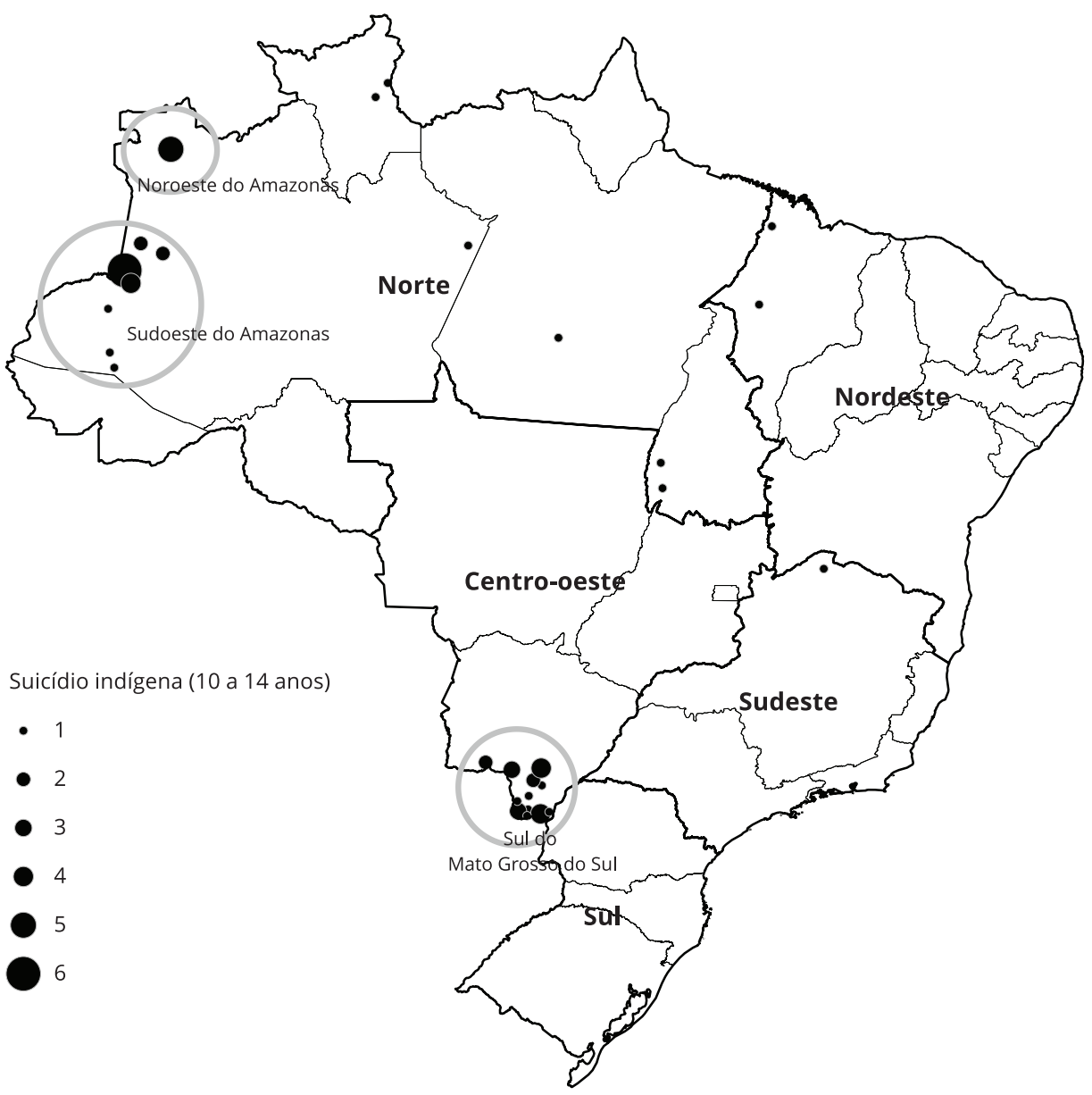




\section{Tabela 3}

Taxas de mortalidade por suicídio entre crianças indígenas e não indígenas. Brasil, 2010-2014.

\begin{tabular}{|c|c|c|c|c|c|c|c|}
\hline & \multicolumn{3}{|c|}{ Indígenas } & \multicolumn{3}{|c|}{ Não indígenas } & \multirow{3}{*}{$\begin{array}{c}\text { RTMS } \\
\text { I/NI (IC95\%) }\end{array}$} \\
\hline & $\mathbf{n}$ & Taxa * (IC95\%) & RTMS & $\mathbf{n}$ & Taxa * (IC95\%) & RTMS & \\
\hline & & & M/F (IC95\%) & & & M/F (IC95\%) & \\
\hline Masculino & 23 & $9,1(6,1-13,7)$ & - & 317 & $0,7(0,6-0,8)$ & - & $13,0(6,5-26,0)$ \\
\hline Feminino & 32 & $12,9(9,1-18,2)$ & - & 212 & $0,5(0,4-0,6)$ & - & $26,7(15,7-45,4)$ \\
\hline Total & 55 & $11,0(8,4-14,3)$ & $0,7(0,1-7,7)$ & 529 & $0,6(0,5-0,6)$ & $1,4(0,3-7,4)$ & $18,5(10,9-31,6)$ \\
\hline
\end{tabular}

F: feminino; I: indígena; IC95\%: intervalo de 95\% de confiança; M: masculino; NI: não indígena; RTMS: razão de taxas de mortalidade por suicídio. Fonte: Sistema de Informações sobre Mortalidade (SIM) do Ministério da Saúde do Brasil (http://tabnet.datasus.gov.br/cgi/deftohtm.exe?sim/ cnv/ext10uf.def, acessado em 18/Dez/2018); Instituto Brasileiro de Geografia e Estatística (https://sidra.ibge.gov.br/pesquisa/censo-demografico/ demografico-2010/inicial, acessado em 18/Dez/2018).

* Por 100.000 .

\section{Discussão}

Este é o primeiro artigo que analisa, em escala nacional, a mortalidade por suicídio entre crianças indígenas no Brasil. Por meio deste estudo, podem ser evidenciadas algumas especificidades das características do suicídio em crianças indígenas, sua concentração em determinados municípios e sua maior magnitude, quando comparado em crianças não indígenas.

O enforcamento foi o método mais utilizado tanto entre indígenas como não indígenas. Evidências apontam que o enforcamento consiste no principal método utilizado por crianças 1,4 , e que haveria uma tendência a diversificação dos métodos com o avançar da idade 4,5,17. No Brasil, o enforcamento foi utilizado mais frequentemente entre indígenas do que entre não indígenas, não se observando, no primeiro grupo, suicídios por intoxicação ou por armas de fogo. Essa diferença não foi observada por Soole et al. 6 entre crianças indígenas e não indígenas australianas, que utilizaram o enforcamento em proporções muito altas e próximas, 95,2\% e 95,8\%, respectivamente. Ademais, no nosso estudo, não se observou diferença na proporção de utilização do enforcamento entre meninos e meninas indígenas, enquanto meninos não indígenas utilizaram mais desse método do que as meninas. Meninos não indígenas utilizaram, ainda, mais frequentemente do que meninas não indígenas armas de fogo, enquanto elas se utilizaram mais do que eles da intoxicação. Esse padrão de utilização de métodos mais letais (enforcamento e arma de fogo) entre meninos foi observado na Argentina 18, na Áustria 19 e numa revisão que se valeu de dados de 101 países 16 . A utilização do enforcamento como método preferencial por crianças indígenas, observada neste estudo, independentemente do gênero, além de ser uma especificidade étnico-racial, consiste num desafio à prevenção, pois trata-se de um método altamente letal, possível de ser efetuado utilizando materiais disponíveis em casa. Por outro lado, existe um acúmulo de achados que evidenciam a eficácia da restrição do acesso a armas de fogo e a substâncias capazes de causar morte por envenenamento na prevenção do suicídio em crianças 16 .

Em relação ao local onde ocorreu o óbito, observou-se que, tanto entre indígenas como entre não indígenas, houve predomínio de mortes no domicílio, sem diferença estatística entre os grupos. Tal achado replica o observado em estudos prévios sobre suicídio entre crianças ${ }^{4}$. No nosso estudo, a maior parte dos óbitos ocorreu por enforcamento, método altamente letal, que tenderia a causar morte instantânea em muitos casos, sendo o atendimento hospitalar menos frequente e reservado àqueles que tiveram uma célere remoção para o ambiente nosocomial. Por outro lado, as crianças indígenas morreram menos frequentemente em ambiente hospitalar, comparando-se às não indígenas. Tal fato já foi observado em estudos nacionais que investigaram o suicídio entre populações indígenas comparativamente às não indígenas, mas não exclusivamente na faixa etária infantil, sendo entendido como algo associado à maior dificuldade de acesso da população nativa a serviços hospitalares 10,11,12. Tal explicação parece ser extensível à população indígena infantil. Por outro lado, nossos achados diferem, em parte, do observado por Soole et al. 6, que, na Austrália, observaram que, embora indígenas 
e não indígenas tenham morrido por suicídio mais frequentemente em domicílio, a proporção foi estatisticamente maior no segundo grupo. Infelizmente, no artigo, os autores não fazem qualquer discussão sobre esse achado. Um último destaque sobre o local da ocorrência do óbito remete para outra especificidade de gênero. Enquanto não se observou diferença na proporção de óbitos em ambiente hospitalar entre meninas e meninos indígenas, verificou-se que, entre não indígenas, meninas morreram mais do que meninos nesse local. Considerando que não haveria diferença no acesso aos serviços hospitalares entre meninos e meninas indígenas, uma possível explicação para esse achado seria a maior ocorrência, entre elas, conforme já discutido, de métodos menos letais, como a intoxicação, que permitiriam maior chance de atendimento em serviços de saúde.

Mais de $90 \%$ dos suicídios entre indígenas se concentraram nas macrorregiões Norte e Centro-oeste, enquanto, nessas áreas, residiam, aproximadamente, 59\% das crianças indígenas do país. Já na macrorregião Nordeste, ocorreram menos de $4 \%$ dos suicídios infantis entre indígenas, enquanto lá reside, aproximadamente, 1/4 das crianças indígenas do Brasil. Ademais, 3/4 dos suicídios entre crianças indígenas ocorreram em um conjunto de 17 municípios, cuja população de crianças indígenas equivale a $16,9 \%$ da nacional 15 . Esses achados em conjunto apontam que não é possível associar diretamente a frequência de suicídios entre crianças indígenas tão somente à distribuição dessa população no território nacional.

É importante destacar que os 17 municípios nos quais se concentraram a maior parte dos suicídios indígenas infantis podem ser agrupados em três grupos: sudoeste do Amazonas, noroeste do Amazonas e sul do Mato Grosso do Sul. Há um acúmulo de trabalhos nacionais que evidenciam que o suicídio entre indígenas é um importante e complexo problema social e de saúde pública em municípios dessas áreas 8,10,11,13,14,20. Assim, os suicídios indígenas infantis concentram-se, especialmente, em áreas nas quais o suicídio consiste em um problema na população nativa em geral, não podendo ser, a princípio, considerado uma questão isolada.

Embora essas áreas tenham em comum a alta ocorrência de suicídio na população indígena, os motivos que vêm sendo recrutados, pela literatura, para compreender esse fenômeno são, de certo modo, distintos, o que, de certa forma, é razoável, visto que trata de contextos diferentes. Destaco que as reflexões que existem na literatura versam sobre o suicídio entre indígenas em geral, e não, de modo específico, sobre o suicídio entre crianças, questão que estamos levantando com certo ineditismo neste artigo.

O suicídio entre indígenas na região sul do Mato Grosso do Sul vem sendo associado à escassez de terras nas quais os indígenas possam reproduzir seus meios tradicionais de vida, associada a diferentes formas de violência e tensões com setores do próspero agronegócio que se desenvolve na região 11,14,20. Por outro lado, tanto no noroeste quanto no sudoeste do Amazonas, encontram-se extensas terras indígenas demarcadas, dentre as maiores do país. Nos últimos 30 anos, essas duas áreas foram alvo de um conjunto de ações governamentais que visavam incrementar a presença do estado brasileiro nesses locais, resultando em grande afluxo de não indígenas para a região e num processo de urbanização da população indígena, sobretudo nos centros regionais ou sub-regionais. Dentre os rearranjos que teriam resultado desse processo, vêm sendo destacados o incremento do consumo de álcool e dificuldades no gerenciamento de conflitos entre gerações, que estariam, de um modo complexo, interagindo, explicando, pelo menos em parte, as elevadas taxas de mortalidade por suicídio entre indígenas nessas áreas 21 . Especificamente em relação ao suicídio de jovens indígenas residentes em São Gabriel da Cachoeira, município localizado no noroeste do Amazonas, Souza \& Orellana 8 apresentaram algumas hipóteses para as elevadas taxas de mortalidade por suicídio. Entre elas, destacaram o afastamento dos jovens das atividades tradicionais de subsistência, bem como as elevadas expectativas juvenis quanto ao futuro após a conclusão do ensino médio que contrastam com uma realidade na qual a possibilidade de continuar estudando e/ou de ter acesso a uma vaga no escasso mercado de trabalho regional é uma possibilidade virtual para a maioria.

Assim, ter terras demarcadas em área suficiente é, sem dúvida, um fator importante de bem-estar coletivo e produtor de saúde para as populações indígenas. Porém, tê-las, por si só, não esgota os desafios de sobrevivência dos povos indígenas. As relações de subalternização com o estado brasileiro e com os demais grupos de interesses político-econômicos que circundam ou adentram legal ou ilegalmente as terras indígenas são mantidas, mesmo que indiretamente, impactando deleteriamente, na maioria das vezes, no modo de vida dos povos indígenas. 
A taxa de mortalidade por suicídio entre crianças no Brasil foi de 0,7/100 mil (0,6-0,7), valor igual ao observado por Lovisi et al. 22, no período de 2004-2006, apontando para uma estabilidade da magnitude do fenômeno ao longo desses anos. O valor encontrado foi igual ao observado na Noruega no período de 2006-2009 23, e menor do que o registrado nos Estados Unidos em 2004, 1,3/100 mil 24, sendo próximo da estimativa da taxa mundial para essa faixa etária que foi de 0,6/100 mil ${ }^{17}$. Por outro lado, esse valor relativamente baixo oculta a elevada taxa de mortalidade por suicídio encontrada entre crianças indígenas no Brasil, de 11,0/100 mil (8,4-14,3), 18,5 (10,9-31,6) vezes maior do que a observada entre não indígenas. A taxa aqui encontrada se aproxima da observada por Soole et al. 6 entre crianças indígenas da Austrália, 10,1/100 mil, que foi 12,6 vezes maior do que a dos não indígenas. Importante destacar, como já discutido, que, como o suicídio entre crianças indígenas não é homogeneamente distribuído no país, existem áreas nas quais essas taxas podem ser ainda maiores. Infelizmente, como o suicídio indígena apresenta taxas mais elevadas na população jovem (de 15 a 24 anos) 7 , os estudos nacionais já realizados raramente apresentam dados sobre suicídio entre crianças indígenas. Uma exceção é o trabalho de Ferreira et al. 20 que informa que, no período de 2004-2006, a taxa de suicídio entre crianças atendidas pelo Distrito Sanitário Especial Indígena do Mato Grosso do Sul foi de 108,6/100 mil.

Possíveis explicações para as mais elevadas taxas de mortalidade por suicídio entre crianças indígenas podem ser buscadas na eventual maior ocorrência, nesse grupo, de fatores de risco para o suicídio próprios dessa faixa etária. Ter um familiar que morreu por suicídio é reconhecido como um importante fator de risco para o suicídio entre crianças ${ }^{4}$. Conforme já apontado, a maior parte dos casos de suicídios entre crianças indígenas ocorreu em locais nos quais o suicídio é um importante problema de saúde pública, em outras faixas etárias entre indígenas. Orellana et al. 13 demonstraram que 17,2\% dos casos de suicídio entre indígenas em Tabatinga, município localizado no sudoeste do Amazonas, ocorreu entre parentes de até segundo grau. Mais especificamente entre crianças indígenas, Lazzarini et al. 14 demonstraram que, na região de Dourados no Mato Grosso do Sul, aproximadamente, metade dos casos de suicídio entre indígenas com menos de 15 anos ocorreu em clusters domiciliares, sendo esse grupo aquele etário no qual esse fenômeno ocorreu mais frequentemente. Ademais, merece destaque o fato que os conceitos indígenas de parentesco são bem mais ampliados e difusos que os utilizados pelos autores antes citados 25,26. Assim, a hipótese que se levanta é que o grupo de criança indígenas ao ter maior probabilidade de possuir parentes que morreram por suicídio estariam em maior risco para morrer por essa mesma causa de óbito, do que crianças não indígenas, o que poderiam auxiliar a explicar, em parte, as diferenças observadas nas taxas de mortalidade.

Existe uma grande diversidade de outros fatores de risco para o suicídio entre crianças, dentre os quais, destacam-se: conflitos familiares, problemas relacionados ao uso de álcool, problemas na escola e abuso infantil 1,4. Estudo qualitativo utilizando narrativas evidenciou a importância do conflito com os pais, em particular as dificuldades de diálogo, como chave explicativa no discurso nativo para o suicídio entre jovens indígenas na região do Alto Rio Negro, no noroeste do Estado do Amazonas 27. Outros estudos propõem, por meio de um proxy (proporção de óbitos ocorridos em dias de fim de semana), uma maior associação entre suicídio e consumo de álcool entre indígenas, em comparação com não indígenas 10,11. Dados de um inquérito nacional sobre saúde escolar evidenciou que os meninos que se autodeclararam indígenas foram aqueles que mais relataram sofrer bullying na escola 28 , e indígenas, em geral, foram os mais propensos a relatarem sofrer violência doméstica 29 . Ainda que essas pesquisas não tenham focado especificamente na faixa etária de 10 a 14 anos, elas abrem possibilidades para investigações e intervenções para o enfrentamento do suicídio entre crianças indígenas no Brasil.

Por outro lado, fenômenos complexos como o suicídio de crianças indígenas, em particular suas elevadas taxas, não podem ser pensados de forma exclusivamente disciplinar. Além de se investigar a desigual ocorrência de certos fatores de risco, faz-se necessário pesquisar, de forma aprofundada, como os próprios indígenas dão sentido a esse fenômeno, como ancoram, no seu universo simbólico, a morte por suicídio, em geral, e a morte por suicídio de crianças, em particular, em termos êmicos. Um ponto importante a ser investigado seria como se configuram, contemporaneamente, na perspectiva indígena, as relações e os eventuais conflitos entre as diferentes gerações. Um delineamento dessa questão poderia auxiliar, por exemplo, na melhor compreensão do que significaria, no contexto indígena, as ideias de conflitos familiares e abuso infantil, tidas, como anteriormente apresentado, 
como fatores de risco para suicídio entre crianças, mas cuja transposição para o universo conceitual indígena tem possivelmente um conjunto de desafios.

Outro importante aspecto ainda a se destacar é o fato de não encontrarmos diferenças nas taxas de mortalidade entre meninos e meninos, nem entre indígenas nem entre não indígenas, de modo semelhante ao observado por Soole et al. 6 na Austrália. A literatura aponta que a razão entre as taxas masculino/feminino tende a aumentar com a idade, principalmente pelo aumento da prevalência de fatores de risco associados ao suicídio, tais como problemas relacionados ao uso de álcool e outras drogas e outros transtornos mentais, que sofre um incremento importante a partir da adolescência, de forma mais importante no sexo masculino 4 . Entretanto, alguns autores apontam taxas mais elevadas já entre meninos em comparação a meninas. Em um estudo envolvendo 101 países, estimou-se uma razão de taxas masculino/feminino de 1,6 17, e, em um estudo na Áustria, essa razão foi estimada em 3,0 19. Infelizmente, nenhum desses estudos apresentou o intervalo de confiança dessas razões, o que limita avaliarmos se há ou não diferença estatística entre as taxas. Especificamente em relação a populações indígenas brasileiras, Lazzarini et al. 14 demonstraram as altas taxas de mortalidade por suicídio entre crianças indígenas residentes na região de Dourados no sul do Mato Grosso do Sul, destacando-se que não havia diferença estatística entre meninos e meninas. Neste mesmo trabalho, as taxas mais altas encontradas no sexo feminino foram justamente entre as meninas de 10 a 14 anos, fato já previamente destacado por Orellana et al. ${ }^{11}$ para meninas indígenas de todo o Estado do Mato Grosso do Sul. Assim, diferentemente do observado em outras faixas etárias, entre crianças, particularmente entre indígenas, o suicídio tenderia a ocorrer em igual magnitude em ambos os sexos, o que representa uma outra especificidade de gênero, na medida em que há uma relativamente maior participação feminina no fenômeno.

Entre as limitações deste trabalho, podemos citar, inicialmente, eventuais problemas de subnotificação e erros de classificação das causas de óbito, algo reconhecidamente problemático quando se fala de suicídios infantis 1. Importante destacar que, no Brasil, houve importantes avanços e melhoria da qualidade dos registros vitais nos últimos anos. Entretanto, em municípios rurais e remotos, principalmente nos com dificuldades de acesso à assistência à saúde e com grandes dimensões, a qualidade dos registros, sobretudo a dos óbitos infantis, é mais baixa, o que demandaria, segundo alguns autores, a necessidade de estratégias de correção para compensar os sub-registros 30 . A não utilização de fatores de correção, sobretudo se consideramos que a maior parte dos municípios nos quais se concentrou os suicídios de crianças indígenas possui características análogas às anteriormente citadas, consiste em uma limitação adicional deste trabalho. Ademais, a subnotificação de suicídios entre indígenas é fato já demonstrado na literatura nacional 13,31. De qualquer modo, a magnitude da mortalidade por suicídio entre crianças indígenas deve até ser maior do que a aqui apresentada.

Outra questão refere-se à dificuldade de transposição de conceitos do universo ocidental para o indígena ${ }^{32}$, tais como os próprios conceitos de suicídio, algo que já discutimos em publicação anterior, como o de infância. Definir infância como um intervalo etário específico, embora estando em consonância com a literatura, e sendo operacionalmente necessário, é algo com certo grau de arbitrariedade. Ademais, em universos culturais distintos, as diferentes fases da vida podem se configurar de modos próprios, como evidenciamos anteriormente noutra pesquisa, sobre juventude no contexto indígena do noroeste amazônico 31 . Ter entre 10 e 14 anos, certamente, não é a mesma coisa entre indígenas e não indígenas e, possivelmente, não será a mesma coisa entre os mais de 300 grupos indígenas brasileiros. A despeito dessas limitações, o trabalho aqui apresentado pôde lançar um primeiro olhar exploratório para o suicídio entre crianças indígenas no Brasil, um tema que vem sendo negligenciado.

Por fim, dentre os achados deste trabalho, destaca-se que as taxas de mortalidade por suicídio entre crianças indígenas são elevadas em comparação as das não indígenas, afetando, de modo igual, meninos e meninas. Cerca de 3/4 dos suicídios de crianças indígenas concentraram-se em 17 municípios, localizados em áreas nas quais se sabe, a partir da literatura, que o suicídio é um importante problema para a população indígena em geral. Tem-se, portanto, a possibilidade de eleger essas áreas como prioritárias para intervenções voltadas para o enfrentamento do suicídio entre indígenas, incluindo crianças. Ademais, depreende-se que o suicídio indígena infantil não deve ser considerado em separado do suicídio indígena em geral, e que medidas culturalmente sensíveis para a redução de vulnerabilidades, para a promoção da saúde e para a prevenção de agravos devem ser implementa- 
das visando ao enfrentamento da questão como um todo. Porém, isso não significa que estratégias direcionadas especificamente para o enfrentamento do suicídio infantil entre indígenas não devam ser implementadas. Dentre tais medidas, destaca-se a potencialidade daquelas que possam concorrer para a prevenção da violência contra a criança, para o combate ao bullying nas escolas, para a redução do consumo e dos danos relacionados ao uso do álcool, bem para a postergação da idade do início do consumo, assim como para qualificar as estratégias comunicativas entre gerações.

\section{Informação adicional}

ORCID: Maximiliano Loiola Ponte de Souza (00000002-4373-7016).

\section{Agradecimento}

Agradeço à Fernanda Fonseca, do Instituto Leônidas e Maria Deane/Fundação Oswaldo Cruz, pela construção da Figura 1.

\section{Referências}

1. Sousa GS, Santos MSP, Silva ATP, Perrilli JGA, Sougey EB. Revisão de literatura sobre suicídio na infância. Ciênc Saúde Colet 2017; 22:3099110.

2. Souza MLP, Ferreira LO. Jurupari se suicidou? Notas para investigação do suicídio no contexto indígena. Saúde Soc 2014; 23:1064-76.

3. Gesell A, Ilg FL, Ames LB. El niño de 5 a 10 años. Buenos Aires: Paidós; 1971.

4. Soole R, Kolves K, De Leo D. Suicide in children: a systematic review. Arch Suicide Res 2015; 19:285-304.

5. Kolves K, De Leo D. Child, adolescent and young adult suicides: a comparison based on the Queensland Suicide Registry. J Child Adolesc Behav 2015; 3:209.

6. Soole R, Kolves K, De Leo D. Suicides in Aboriginal and Torres Strait Islander children: analysis of Queensland Suicide Register. Aust N Z J Public Health 2014; 38:574-8.

7. Pollock NJ, Naicker K, Loro A, Mulay S4, Colman I. Global incidence of suicide among indigenous people: a systematic review. BMC Med 2018; 16:145.

8. Souza MLP, Orellana JDY. Suicide mortality in São Gabriel da Cachoeira, a predominantly indigenous Brazilian municipality. Rev Bras Psiquiatr 2012; 34:34-7.

9. Souza MLP, Orellana JDY. Suicide among the indigenous people in Brazil: a hidden public health issue. Rev Bras Psiquiatr 2012; 34:48990.

10. Souza MLP, Orellana JDY. Desigualdades na mortalidade por suicídio entre indígenas e não indígenas no Estado do Amazonas, Brasil. J Bras Psiquiatr 2013; 62:245-52.

11. Orellana JD, Balieiro AA, Fonseca FR, Basta PC, Souza MLP. Spatial-temporal trends and risk of suicide in Central Brazil: an ecological study contrasting indigenous and non-indigenous populations. Rev Bras Psiquiatr 2016; 38:222-30. 
12. Souza MLP, Onety Júnior RTS. Caracterização da mortalidade por suicídio entre indígenas e não indígenas em Roraima, Brasil, 2009-2013. Epidemiol Serv Saúde 2017; 26:887-93.

13. Orellana JD, Souza CC, Souza MLP. Hidden suicides of the indigenous people of the Brazilian Amazon: gender, alcohol and familial clustering. Rev Colomb Psiquiatr 2018; doi: 10.1016/j.rcp.2017.12.001.

14. Lazzarini TA, Gonçalves CCM, Benites WM, Silva LF, Tsuha DH, Ko AI, et al. Suicide in Brazilian indigenous communities: clustering of cases in children and adolescents by household. Rev Saúde Pública 2018; 52:56.

15. Instituto Brasileiro de Geografia e Estatística. Os indígenas no censo demográfico 2010: primeiras considerações com base no quesito cor ou raça. Rio de Janeiro: Instituto Brasileiro de Geografia e Estatística; 2012.

16. Organização Mundial da Saúde. Classificação estatística internacional de doenças e problemas relacionados à saúde (CID-10). 10a Rev. 8a Ed. São Paulo: Universidade de São Paulo; 2000.

17. Kolves K, De Leo D. Suicide methods in children and adolescents. Eur Child Adolesc Psychiatry 2017; 26:155-64.

18. Bella ME, Acosta L, Villacé B, López de Neira M, Enders J, Fernandez R. Analysis of mortality from suicide in children, adolescents and youth. Argentina, 2005-2007. Arch Argent Pediatr 2013; 111:16-21.

19. Dervic K, Friedrich E, Oquendo MA, Voracek M, Friedrich MH, Sonneck G. Suicide in Austrian children and young adolescentes aged 14 and youger. Eur Child Adolesc Psychiatry 2006; 15:427-34

20. Ferreira MEV, Matsuo T, Souza RKT. Aspectos demográficos e mortalidade de populações indígenas do Estado do Mato Grosso do Sul, Brasil. Cad Saúde Pública 2011; 27:2327-39.

21. Orellana JD, Basta PC, Souza MLP. Mortality by suicide: a focus on municipalities with a high proportion of self-reported indigenous people in the state of Amazonas, Brazil. Rev Bras Epidemiol 2013; 16:659-69.
22. Lovisi GM, Santos SA, Legay L, Abelha L, Valencia E. Análise epidemiológica do suicídio no Brasil entre 1980 e 2006. Rev Bras Psiquiatr 2009; 31 Suppl 2:S86-93.

23. Dervic K, Brent DA, Oquendo MA. Completed suicide in childhood. Psychiatr Clin North Am 2008; 31:271-91.

24. Kloos AL, Collins R, Weller RA, Weller EB. Suicide in preadolescents: who is at risk? Curr Psychiatry Rep 2007; 9:89-93.

25. Segger A, da Matta R, Viveiros de Castro E. A construção da pessoa nas sociedades indígenas brasileiras. Boletim do Museu Nacional 1979; 32:2-19.

26. Viveiros de Castro E. A inconstância da alma selvagem. São Paulo: Cosac Naify; 2002.

27. Souza MLP. Narrativas indígenas sobre suicídio no Alto Rio Negro, Brasil: tecendo sentidos. Saúde Soc 2016; 25:145-59.

28. Silva JL, Mello FCM, Oliveira WA, Prado RR, Silva MAI, Malta DC. Vitimização por bullying em estudantes brasileiros: resultados da Pesquisa Nacional de Saúde do Escolar (PENSE). Texto Contexto Enferm 2018; 27:e0310017.

29. Malta DC, Stopa SR, Santos MAS, Andrade SSCA, Oliveira MM, Prado RR, et al. Fatores de risco e proteção de doenças e agravos não transmissíveis em adolescentes segundo raça/ cor: Pesquisa Nacional de Saúde do Escolar. Rev Bras Epidemiol 2017; 20:247-59.

30. Almeida WS, Szwarcwald CL. Adequação das informações de mortalidade e correção dos óbitos informados a partir da Pesquisa de Busca Ativa. Ciênc Saúde Colet 2017; 22:3193203.

31. Souza MLP, Orellana JDY. Qualidade do registro de óbitos por suicídio em município amazônico com alta proporção de autodeclarados indígenas. In: Duran DM, Souza JH, organizadores. Suicídio: diálogos interdisciplinares. Manaus: EDUA; 2018. p. 75-94.

32. Souza MLP, Deslandes SF, Garnelo L. Histórias-míticas e construção da pessoa: ambiguidade dos corpos e juventude indígena em um contexto de transformações. Ciênc Saúde Colet $2011 ; 16: 401-10$. 


\section{Abstract}

This study aimed to describe the characteristics, distribution, and mortality rates from suicide in indigenous children in Brazil compared to nonindigenous children. This descriptive study covered the years from 2010 to 2014, using national databases. The study collected deaths in individuals 10 to 14 years of age whose underlying cause was "inentional self-inflicted injury". Hanging was the most frequently used means in both indigenous and non-indigenous children, although it was more frequent in the former. Among indigenous children, suicides in hospitals or other healthcare establishments were less common than in non-indigenous. Approximately three-fourths of suicides in indigenous children occurred in just 17 municipalities. The mortality rate from suicide among indigenous children was 11.0/100,000 (8.4-14.3), or 18.5 times higher (10.9-31.6) than in non-indigenous, which was 0.6/100,000 (0.5-0.6), with no differences between boys and girls. This study showed for the first time on a national scale the specific characteristics of suicide in indigenous children, with high rates, and also identified priority areas for interventions.

Suicide; Child; Indigenous Population

\section{Resumen}

El objetivo de este trabajo fue describir las características, distribución y tasas de mortalidad por suicidio entre niños indígenas en Brasil, en comparación con los no indígenas. Se realizó un estudio descriptivo, desde el año 2010 hasta el 2014, utilizando datos de los sistemas nacionales de información. Se seleccionaron los fallecimientos de personas con edades comprendidas de 10 a 14 años, cuya causa básica de fallecimiento se clasificó como "lesiones auto provocadas voluntariamente". El ahorcamiento fue el medio utilizado con más frecuencia, tanto entre indígenas como entre no indígenas, aunque haya sido más frecuente en el primer grupo. Entre indígenas, la ocurrencia de suicidios en hospitales u otros establecimientos de salud fue menor de lo que se observó entre los no indígenas. Aproximadamente, 3/4 de los suicidios entre niños indigenas se produjeron en 17 municipios. La tasa de mortalidad por suicidio entre niños indígenas fue de 11,0/100 mil (8,4-14,3), 18,5 $(10,9-31,6)$ veces mayor que la observada entre los no indígenas, 0,6/100 mil (0,5-0,6), sin diferencias entre niños y niñas. Este trabajo evidenció, por primera vez, en escala nacional, especificidades de las características del suicidio indígena, sus elevadas tasas, así como, incluso, se identificaron áreas prioritarias para intervenciones.

Suicidio; Niño; Población Indígena
Recebido em 31/Jan/2019

Versão final reapresentada em 26/Mar/2019

Aprovado em 17/Abr/2019 